\title{
A Fractal Measure for Comparing the Work Effort of Human and Artificial Agents Performing Management Functions
}

\author{
Matthew E. Gladden \\ Georgetown University, Washington, DC, USA; NeuraXenetica LLC, Indianapolis, IN, USA \\ Email: matthew.e.gladden@gmail.com
}

\begin{abstract}
Thanks to the growing sophistication of artificial agent technologies, businesses will increasingly face decisions of whether to have a human employee or artificial agent perform a particular function. This makes it desirable to have a common temporal measure for comparing the work effort that human beings and artificial agents can apply to a role. Existing temporal measures of work effort are formulated to apply either to human employees (e.g., FTE and billable hours) or computer-based systems (e.g., mean time to failure and availability) but not both. In this paper we propose a new temporal measure of work effort based on fractal dimension that applies equally to the work of human beings and artificial agents performing management functions. We then consider four potential cases to demonstrate the measure's diagnostic value in assessing strengths (e.g., flexibility) and risks (e.g., switch costs) reflected by the temporal work dynamics of particular managers.
\end{abstract}

\section{The Need for a Common Temporal Measure of Work} EFFORT

$\mathrm{T}$ HE increasing power and sophistication of artificial agent technology is allowing businesses to employ artificial agents in a growing number of roles. Artificial agents are no longer restricted simply to performing logistical functions such as resource scheduling, but are now capable of more complex interpersonal workplace behavior such as using social intelligence to effectively manage the limitations, abilities, and expectations of human employees [1], recognizing and manifesting culture-specific behaviors in interactions with human colleagues [2], and assessing the performance of human members of virtual teams [3]. It is thus gradually becoming more feasible to design artificial agents capable of performing the four key functions carried out by human managers, which are planning, organizing, leading, and controlling [4].

As a result of such recent and anticipated future advances, businesses will increasingly be faced with concrete decisions about whether, for example, the manager of a new corporate call center should be an experienced human manager or the latest artificial agent system. Such decisions will be shaped by a large number of strategic, financial, technological, political, legal, ethical, and operational factors. One particular element to be taken into account is that of temporal work effort: i.e., how much time would a human manager actually be able to dedicate to carrying out the necessary work functions, given the fact that physiological, cultural, legal, and ethical constraints limit the number of hours per week that a human being is capable of working? Similarly, how much time would an artificial agent be able to dedicate to carrying out the necessary work functions, given the fact that scheduled maintenance or unscheduled outages can limit the uptime of computer-based systems? Knowing how much time per day (or week, or other relevant time interval) a manager will be available to carry out his or her functions of planning, organizing, leading, and controlling becomes especially relevant in an interconnected age when global businesses operate around the clock, and managers are expected to be available to respond to inquiries and make decisions at almost any time of the night or day.

In the case of human professionals, temporal measures such as 'full-time equivalent' (FTE) [5] and 'billable hours' are often used to quantify one's work effort. Computerbased systems, meanwhile, often use temporal measures such as 'availability' and 'reliability.' In the following sections, we will analyze such existing measures and then develop a new fractal-dimension-based temporal measure for work effort that has at least two notable advantages: it is applicable to the work effort of both human and artificial agent managers, and it provides valuable diagnostic insights into the strengths and dangers of an individual manager's temporal work dynamics that are not provided by existing measures.

\section{MeAsures of Work EFForT FOR COMPUTER-BASED SYSTEMS}

\section{A. Availability and Reliability}

A computer's reliability is often quantified as the mean time to failure (MTTF), the average length of time that a system will remain continuously in operation before experiencing its next failure [6]. The mean time to repair (MTTR) is the average length of time needed to detect and repair the failure and return the system to operation. A computer's steady-state availability $A$ is the likelihood that the computer is operating at a particular moment, and is related to MTTF and MTTR in the equation [6]:

$$
A=\frac{\mathrm{MTTF}}{\mathrm{MTTF}+\mathrm{MTTR}}
$$

A standard requirement for commercial computer systems is $99.99 \%$ availability over the course of a year [7].

Availability has traditionally been understood in a binary manner: a system is either 'up' or 'down.' Rossebeø et al. argue that a more sophisticated measure is needed that takes qualitative aspects into account and suggest recognizing a range of intermediate qualitative states between simply 'up' 
and 'down' [8]. As we explain below, the measure proposed in this paper takes a different approach: its unique diagnostic value comes not from adding a qualitative component but from considering more carefully the fineness and resolution of the time-scales on which measurements are being made.

\section{B. Time-scales for Measuring Computer Performance}

A computer performs actions across a vast range of timescales. As Gunther notes, if a typical computer's CPU cycle were 'scaled up' so that it lasted one second, then using that same scale, a DRAM access would take about one minute, a single disk seek would require roughly 1.35 months, and a tape access would more than a century [7]. He explains that when measuring performance, "Only those changes that occur on a timescale similar to the quantity we are trying to predict will have the most impact on its value. All other (i.e., faster) changes in the system can usually be ignored.... In modeling the performance of a database system where the response time is measured in seconds, it would be counterproductive to include all the times for execution of every CPU instruction."

In a business context, artificial agents performing certain logistical or data-analysis tasks can operate at speeds constrained only by the laws of physics and availability of needed resources. However, an artificial agent manager whose role involves planning, organizing, leading, and controlling the activity of human colleagues should have its work effort measured within a corresponding time-scale. Thus for our present purposes there is no need to consider phenomena such as metastability that have major implications for computer design and functionality but are only directly relevant at the smallest temporal scale [7].

Viewed from the microscopic end of the temporal spectrum, the regulator of all activity within a computer system is the 'clock tick' or 'fundamental interval of time' created by an interrupt sent from the system's hardware clock to the operating system's kernel; in a Unix system, this tick interval is often set at $10 \mathrm{~ms}$ [7], during which time roughly $3.2 \times 10^{5} \mathrm{CPU}$ cycles might occur. For an artificial agent system operating on a serial processor architecture, there is no need to adopt a temporal measure capable of resolving each individual $\mathrm{CPU}$ cycle, as that would not provide information that is directly relevant to the tasks in which the agent's work performance will be evaluated and which take place over a much longer time-frame. For example, an artificial agent manager might interact with human colleagues by generating text or images displayed on a screen. Assuming a screen refresh rate of $60 \mathrm{~Hz}$, this yields a single work interval (or frame) of roughly $17 \mathrm{~ms}$. Writing output data to disk would require a minimum work interval of roughly $3.50 \mathrm{~ms}$ for a disk seek [7]. If the artificial agent is generating speech or other audio to be heard by human beings, a standard sampling rate of $48,000 \mathrm{~Hz}$ would yield a single work interval of roughly $0.02 \mathrm{~ms}$.

At the macroscopic end of the time-scale, it is not unknown for servers to run for several years without rebooting or a moment of downtime [9]. If we view an artificial agent manager as a form of enterprise software, we might expect its lifespan to average around 9 years and to be no shorter than 2 years [10]. Thus while a coarser or finer temporal resolution is possible, our proposed temporal measure for work effort should prove sufficient for artificial agent systems as long as it can encompass time-scales ranging from $0.02 \mathrm{~ms}$ up to several years.

\section{Measures of Work Effort for Human Employees}

\section{A. The Significance of a Year as a Temporal Unit}

We can now consider the case of a human manager. In principle, the longest possible macroscopic time-scale of work effort that one can utilize for a human employee is a biological lifespan. In practice, though, the relevant timescale is obviously much shorter. In the United States, a typical managerial employee only remains with his or her current employer for about 5.5 years before moving to a new organization [11]. The 'year' has significant historical and conceptual value as a fundamental measure of human work activity. Just as enterprise system availability is often cited in terms of uptime per operating year, productivity figures for human workers are typically based on an annual timeframe [12].

Having taken the year as our initial frame of reference, how do we quantify the portion of a given year that a human employee actually spends on his or her work? For this purpose, the largest relevant subunit is that of a single week, as professional workers regularly assess a job's fringe benefits according to how many weeks of vacation they receive each year, and government agencies and researchers often track this data. The number of weeks worked per year varies significantly across nations and cultures [13].

\section{B. The Significance of an Hour as a Temporal Unit}

Even if we know that two employees both 'work' the same number of weeks per year, this fact does not yet tell us much about their relative levels of work effort, as it is possible for the employees to differ vastly in how many hours they work each week. Here, too, there is significant variation across nations and cultures and between specific jobs [13]. For example, an American law firm will likely expect attorneys to work more than 50 hours per week [14], while employees of high-tech Silicon Valley firms are routinely expected to work over 100 hours per week when project deadlines are approaching [15].

\section{How Much Work in an Hour of Work?}

The hour, though, is certainly not the smallest quantifiable interval of employee work effort. Two employees may consider themselves to have just spent an hour 'working,' but the number of minutes of work actually performed by each can differ greatly. In some professions, it is common to track work effort in sub-hour intervals. For example, attorneys with law American law firms typically track their work time in six-minute intervals and sometimes record and bill clients for work that took as little as one minute. For every hour that an attorney spends 'at work,' an average of roughly 45 minutes will count toward billable hours [14].

In other professions, employers have given up any attempt at precisely measuring how much time an employee is putting into their work, as the advent of new communications technologies has caused 'work time' and 
'personal time' to meld into an indistinguishable blur [15]. The rise of multitasking and 'continuous partial attention' drives human workers to constantly monitor emails, texts, and instant messages, even while in the middle of meetings or conversations [16]. For knowledge workers, this continual checking of email can consume up to $25 \%$ of their workday [17]. While much of this nonstop communication activity is work-related, the existence of workplace phenomena such as shirking, social loafing, and job neglect means that a significant number of these electronic interruptions do not relate to work at all, but are purely personal. In particular, younger employees of the Millennial generation are less fond of email and tend to prefer text messaging, instant messaging, [18] and other forms of micro-communication that produce shorter but more frequent non-work interruptions to their work activities. Because professional employees can alternate between work-related and personal actions at such a rapid rate (once every few seconds, if not faster), it is now "very hard to tell when people are working and when people are not working," as a Silicon Valley executive reported in Shih's study [15]. In an effort to counteract this constant stream of distractions, some Extreme Programming (XP) teams employ the Pomodoro Technique, a time-boxing strategy in which physical timers are used to enforce a steady pace consisting of 25 minutes of focused work followed by a brief break [19].

\section{D.Identifying a Minimum Time Unit of Work by Human Managers}

Within a given period of 'work,' there may be alternating periods of work and non-work that are measured in seconds, not minutes. However, in attempting to identify the minimum unit of work of which humans are capable, it is valuable to consider time-scales even much smaller than a second. For example, scholars have estimated that the human brain is capable of between $10^{14}$ and $10^{16}$ calculations per second [20], [21], or roughly $6.6 \times 10^{16}$ FLOPS [22], although the massively distributed parallel processing architecture of the brain [21] means that many calculations are taking place simultaneously, and the duration of a single calculation cannot be determined by simply dividing one second by, say, $10^{15}$. In attempting to estimate the duration of a single 'calculation' performed by the brain, scholars have alternately cited the fact that an individual neuron can fire as often as 1,000 times a second [21], that "synapses carry out floating point operations ... at a temporal resolution approaching about $1000 \mathrm{~Hz}$ " [23], that a neuron is capable of firing roughly once every $5 \mathrm{~ms}$ [24], or that the brain operates at a rate of speed of "around 100 cycles per second" [25]. These estimates yield a range of 1-10ms for the brain's smallest temporal unit of work activity.

It is helpful, though, to refer once more to Gunther's position on the measurement of computer performance: we can essentially ignore activity taking place within a system on a time-scale shorter than that of our work-relevant inputs and outputs, as it is "more likely to be part of the background noise rather than the main theme" [7]. In the case of a human being considered qua employee, the firing of a single synapse does not directly constitute 'work.' The work of planning, organizing, leading, and controlling for which human managers are employed typically involves more complicated inputs and outputs such as engaging in conversation or reading and creating documents. The smallest temporal unit of work would be the smallest unit relevant in the performance of such tasks.

That unit appears to be an interval of roughly $50 \mathrm{~ms}$. Studies have shown that if one alternates too quickly between two tasks that require the same cognitive resources, one's performance on both tasks will be negatively impacted [26], as shifting from one mental task to another incurs a 'switch cost' of both a temporal delay and an increased error rate [27], which lowers productivity [28]. In particular, the human brain needs around $120 \mathrm{~ms}$ to fully allocate its attention to a new stimulus [29]. Marchetti cites diverse studies supporting the claim that the minimum 'integration time' needed for the brain to meld disparate sensory input into a conscious perception of a single event or experience is roughly 50-250 ms, with a median of about $100 \mathrm{~ms}$ [30]. These findings make it unlikely that a human manager would be capable of performing individual instances of work that need to be measured using a time-frame shorter than 50 ms. If one attempted to alternate between tasks faster than once every $50 \mathrm{~ms}$, one's brain would not even have time to focus attention on a new task before abandoning it for yet another task.

\section{E. Durations of Particular Work Inputs and Outputs}

This minimum interval of roughly $50 \mathrm{~ms}$ is supported by the fact that the kinds of inputs and outputs that human managers typically utilize when performing work-related functions do not have durations shorter than this interval. For example, Hamilton notes that human beings can think at a rate of 400-800 words per minute, while we typically speak at 100-175 words per minute (with each spoken word comprising an average of 4-5 phonemes [31]). Optimal listening comprehension occurs when a speaker speaks at 275-300 words per minute, which gives a listener's mind less time to become distracted or daydream between each of the speaker's words [32]. Adult native speakers of English typically read 200-250 words per minute [33]. Regarding

\section{TABLE I.}

AVERAGE TIME NEEDED BY THE HUMAN BRAIN TO PERFORM WORKRELATED INPUT, PROCESSING, AND OUTPUT FUNCTIONS

\begin{tabular}{|l|l|}
\hline \multicolumn{1}{|c|}{ Activity } & \multicolumn{1}{|c|}{$\begin{array}{c}\text { Average } \\
\text { Time }\end{array}$} \\
\hline Fully allocating attention to a new stimulus & $120 \mathrm{~ms}$ \\
\hline $\begin{array}{l}\text { Consciously perceiving a single coherent } \\
\text { experience or event }\end{array}$ & $50-250 \mathrm{~ms}$ \\
\hline $\begin{array}{l}\text { Hearing one spoken phoneme } \\
\text { (4.4 phonemes per word) }\end{array}$ & $45-50 \mathrm{~ms}$ \\
\hline Hearing one spoken word & $200-220 \mathrm{~ms}$ \\
\hline Reading one printed word & $200-250 \mathrm{~ms}$ \\
\hline Thinking one word & $75-150 \mathrm{~ms}$ \\
\hline Speaking one phoneme (4.4 phonemes per word) & $20-140 \mathrm{~ms}$ \\
\hline Speaking one word & $90-600 \mathrm{~ms}$ \\
\hline Typing one character (5.5 characters per word) & $\leq 75 \mathrm{~ms}$ \\
\hline Typing one word & $\leq 400 \mathrm{~ms}$ \\
\hline Writing one word in shorthand & $\leq 170 \mathrm{~ms}$ \\
\hline
\end{tabular}


work output, the fastest sustainable typing rate is roughly 150 words per minute [34], with each word comprising an average of 5-6 characters; the fastest known shorthand writing speed is roughly 350 words per minute [35]; and the fastest known human speaker is able to clearly articulate more than 650 words per minute [36]. When these rates are converted into milliseconds, they yield the intervals seen in Table I.

\section{F. The Fractal Self-similarity of Human Work Cycles}

As we have seen, for artificial agent managers, the timescales relevant to their work effort range from several years down to about $10 \mathrm{~ms}$, while for human managers they range from several years down to around $50 \mathrm{~ms}$. Within this range, there are multiple relevant time-scales and activity cycles of different lengths that demonstrate an interesting degree of self-similarity: within a given year of work, a typical human manager will spend many consecutive weeks working, interrupted periodically by non-work weeks of vacation. Within a given week of work, he will spend spans of several consecutive hours working, followed by non-work hours when he is asleep or out of the office. Within a given hour of work, his spans of minutes spent working will be followed by non-work intervals when he is daydreaming or writing a personal email. The roughly self-similar nature of this temporal dynamic opens the door to understanding a human manager's work activity as a fractal time series.

The fractal nature of our typical human work dynamics is not at all surprising: as Longo and Montévil note, fractal-like dynamics are "ubiquitous in biology, ... in particular when we consider processes associated with physiological regulation" [37]. Lloyd notes that when an organism's biological processes operating on multiple time-frames displaying fractal temporal coherence, it creates a scale-free system with "robust yet flexible integrated performance" in which the oscillatory dynamics with long memory allow the organism to predict and respond to long-term environmental conditions such as tidal, seasonal, and annual cycles, while the short-term cycles coordinate internal processes such as organ functioning and cellular division [38].

\section{Calculating the Fractal Dimension of Work Effort}

\section{A. Significance of the Fractal Dimension}

One of the most important and meaningful attributes of a fractal time series is that it possesses a fractal dimension that one can calculate and which captures valuable information about the series' temporal dynamics. The calculation of the fractal dimension of biological phenomena has varied practical applications. For example, analysis of the fractal dimension of EEG data can be used to quantify the level of concentration during mental tasks [39], and fractal analysis has demonstrated that healthy hearts display greater rhythmic complexity than diseased hearts [37].

The fractal dimension of empirically observed natural phenomena can be described by the equation $D=2-H$, where $H$ is the Hurst exponent of the time series as graphed in two-dimensional Cartesian space. In this approach, an xcoordinate is the time at which a value was measured, and the y-coordinate is the value measured at that time [40]. The case $0<H<1 / 2$ represents a dynamic that is variously described as antipersistent, irregular, or trend-reversing: if the value in one moment is greater than the mean, the value in the next consecutive moment is likely to be less than the mean. The case $H=1 / 2$ represents a random-walk process such as Brownian motion, in which the value in the next consecutive moment is equally likely to move toward or away from the mean. The case $1 / 2<H<1$ is described as persistent or quasi-regular: the value at the next consecutive moment in time is likely to be the same as the value in the previous moment [40], [41]. In this case, we can say that the dynamic has long memory.

\section{B. Work Effort as a Time Series of Binary Values}

Graphing a time-series in two-dimensional space is useful for natural phenomena such as earthquakes that occur at different times with different intensities [42]. However, in the case of developing a temporal measure for quantifying the work effort of human and artificial managers, we suggest that a different approach is warranted. Graphing an agent's work effort in two-dimensional space would be useful if the work effort displayed by a human or artificial agent manager at a particular instant of time were able to range across a continuous spectrum of values. However, in this case we have only a binary set of possible values: at any given instant, an agent is either focusing its attention on its work, or it is not. Marchetti draws on research from several areas of psychology to show that the human mind is incapable of dividing its attention between two different scenes, attitudes, or 'observational levels' at the same instant in time. (We would suggest that the same will likely be true for any artificial agent whose cognitive capacities are modeled closely on those of the human brain's neural network, as well as for any artificial agent governed by a computer program in the form of executable code.) As we saw above, the brain's attention mechanism is capable of alternating attention between two different thoughts or scenes with great rapidity (as in cases of so-called 'multitasking'), however in any given instant of time, our attention is allocated to at most one of those thoughts or scenes. This means that work effort cannot be quantified by saying, for example, that "At moment $t, 70 \%$ of the agent's attention was dedicated to its work." Instead, one would say that "For all of the indivisible instances of attention that took place during time interval $[a, b]$, in $70 \%$ of those instances the agent's attention was focused on its work."

Mandelbrot notes that if the fractal dimension of a time series graphed in two-dimensional space is represented by the equation $D=2-H$, then the zero set (or any other level set) of the graphed time series would have fractal dimension [40]:

$$
D=1-H
$$

We can use this equation to relate the fractal dimension and Hurst exponent for work effort when we understand work effort as graphed on a one-dimensional line segment. The length of the entire segment represents the entire time available (such as a year, week, or hour) during which an agent can potentially be performing work. Those instants of actual work form the set that is graphed on the line segment, 
while instants of non-work do not belong to the set. With this binary approach, we can envision the depiction of an agent's work effort across time as a series of instances of work and non-work graphed on a line segment that resembles a generalized Cantor set in which the moments of work are those points contained in the set and moments of non-work are portions of a deleted interval. Because this is a graph of an empirically observed natural phenomenon rather than a purely mathematical object, it would have a minimum fineness and resolution: if our minimum unit of time is 10 $\mathrm{ms}$ and we graph a line segment representing one hour, it would comprise $3.6 \times 10^{6}$ such units of work or non-work.

In this context, the Hurst exponent takes on a different (and perhaps even counterintuitive) meaning. For a twodimensional graph of a time series with $H \approx 0$, successive $y-$ values alternate antipersistently around the mean, and the graphed line fills up a relatively large share of the twodimensional space. For a one-dimensional graph of a binary time series, one might visualize the set as though it contains a single point that is able to slide back and forth along the $\mathrm{x}$ axis to occupy many different $\mathrm{x}$-values simultaneously, thus forming the set. For a set with high persistence $(H \approx 1)$, the point may be locked to a single $\mathrm{x}$-value, reflecting a process with long memory. For a set with low persistence $(H \approx 0)$, the point 'forgets' where it is and is free to move up and down the line segment, occupying many different $\mathrm{x}$-values. This conceptualization reflects the fact that the twodimensional graph of an antipersistent time series will cross the horizontal line determined by the mean y-value at many different places, whereas the graph of a persistent process might only cross it once, and the graph of a random-walk process can intersect it either one or many times.

\section{Formulating our Fractal Measure}

\section{A. Advantages of the Box-Counting Method}

Different methods exist for calculating fractal dimension. A number of scholars prefer the Minkowski-Bouligand or box-counting dimension over alternatives such as the areaperimeter or power spectrum methods for estimating the fractal dimension of natural phenomena as diverse as seismic activity, electrical activity in the brain, and physical surface features at the nanometer scale [42], [39], [43]. Longo and Montévil argue that while it lacks some of the mathematical import found in other definitions of fractal dimension such as the Hausdorff dimension, the boxcounting dimension has an advantage in that it can easily be applied to empirically observed phenomena [37].

In order to develop our comparative fractal measure of work effort for human and artificial agent managers, we have thus employed the box-counting method to estimate the temporal dynamics' fractal dimension. The box-counting dimension $D$ of set $F$ can be calculated as:

$$
D=\lim _{\delta \rightarrow 0} \frac{\log N_{\delta}(F)}{-\log \delta} .
$$

Here $N_{\delta}(F)$ is the smallest number of sets of diameter $\delta$ that cover the set $F$ [44]. When using the box-counting method to estimate the fractal dimension of natural phenomena, this can be done by calculating the average value of $D$ that results when one empirically determines for multiple values of $\delta$ [45].

\section{B. Calculation and Notation of our Fractal Measure}

When we applied this approach to calculate the boxcounting fractal dimension $D$ for the work effort of particular hypothetical human and artificial agent managers, it yielded insights that could be useful for understanding, comparing, and enhancing the temporal work dynamics of such agents.

To accomplish this, we considered an agent's typical work effort as viewed across on three different time-scales or levels: 1) The set $F_{1}$ includes those weeks worked within a span $S_{1}$ of five years (or 260 weeks), for which the covering sets used for the box-counting estimation were $\delta_{\mathrm{a}}=$ 4 weeks, $\delta_{\mathrm{b}}=2$ weeks, and $\delta_{\mathrm{c}}=1$ week. 2) The set $F_{2}$ includes those hours worked within a span $S_{2}$ of one week (or 168 hours), for which the covering sets used for the boxcounting estimation were $\delta_{\mathrm{a}}=4$ hours, $\delta_{\mathrm{b}}=2$ hours, and $\delta_{\mathrm{c}}=$ 1 hour. 3) The set $F_{3}$ includes those minutes worked within a span $S_{3}$ of one hour, for which the covering sets used for the box-counting estimation were $\delta_{\mathrm{a}}=1$ minute, $\delta_{\mathrm{b}}=30$ seconds, and $\delta_{\mathrm{c}}=15$ seconds. Using the box-counting method, we calculated $D_{1}, D_{2}$, and $D_{3}$ for the time-scales $F_{1}$, $F_{2}$, and $F_{3}$, respectively, and averaged those values to produce a mean value of $D=\left(D_{1}, D_{2}, D_{3}\right)$ for a particular agent. We then calculated the estimated value for the Hurst exponent for that agent's temporal dynamic with the equation $H=1-D$.

Drawing on the data considered in previous sections for the typical temporal performance of human professionals and artificial agents (envisioned as hardware and software systems), we present four specific hypothetical cases and the values of $D$ and $H$ calculated for each.

\section{Vi. Applying our Measure to Particular Cases}

\section{A. Temporal Dynamics of Human Manager A}

Consider a hypothetical Human Manager A whose work effort approaches the maximum of which contemporary human beings are capable. This manager does not take any weeks of vacation during the five years worked in his position ( $S_{1}=260$ weeks, $N_{\delta}\left(F_{1}\right)=260$ weeks). He concentrates exclusively on his career, working an average of 90 hours per week ( $S_{2}=168$ hours, $N_{\delta}\left(F_{2}\right)=90$ hours $)$. During the work day, he avoids all possible distractions and, relying on an approximation of the Pomodoro Technique, spends only 5 minutes of each 'work hour' not performing work-related functions $\left(S_{3}=60\right.$ minutes, $N_{\delta}\left(F_{3}\right)=55$ minutes). We graphed each of these situations on a line segment that we then considered at three different temporal resolutions. Within the graph of the time series, a moment of work is indicated with a colored vertical slice, and a moment of non-work is indicated with an unshaded interval. A graph of the temporal work dynamics of Human Manager A is seen in Fig. 1 below. For an agent with these characteristics, we have calculated $D=0.962, H=0.038$, and availability (understood as the likelihood that any randomly-selected 


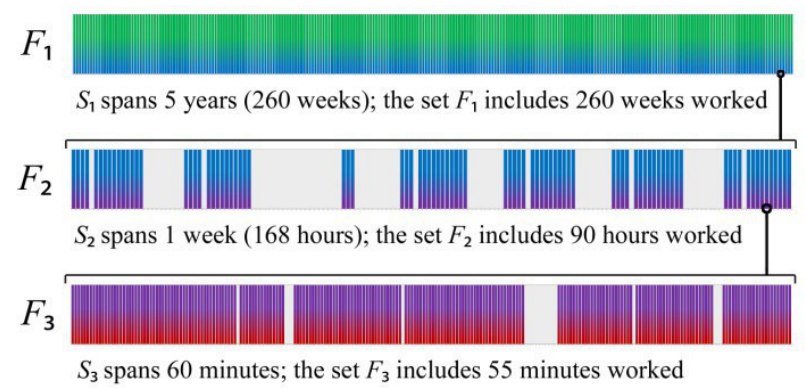

Fig. 1: Human Manager A's periods of work and non-work

instant of time will fall during a moment of work rather than non-work) as $A=49.1 \%$.

\section{B. Temporal Dynamics of Human Manager B}

Hypothetical Human Manager B represents the opposite end of the spectrum: his time commitment approaches the lowest amount possible for someone who is fulfilling a management role with an organization. We suppose that Human Manager B spends only half of the weeks in the year working $\left(S_{1}=260\right.$ weeks, $N_{\delta}\left(F_{1}\right)=130$ weeks). Even during those weeks when he is working, the manager dedicates only 10 hours of effort to this particular position ( $S_{2}=168$ hours, $N_{\delta}\left(F_{2}\right)=10$ hours). Moreover, during each hour of 'work,' the manager spends only a third of the time focused directly on work-related tasks, with the rest of the time representing distractions or non-work-related activities ( $S_{3}=60$ minutes, $N_{\delta}\left(F_{3}\right)=20$ minutes). A graph of the temporal work dynamics of Human Manager B is seen in Fig. 2 below. For an agent with these characteristics, we have calculated $D=0.532, H=0.468$, and $A=1.0 \%$.

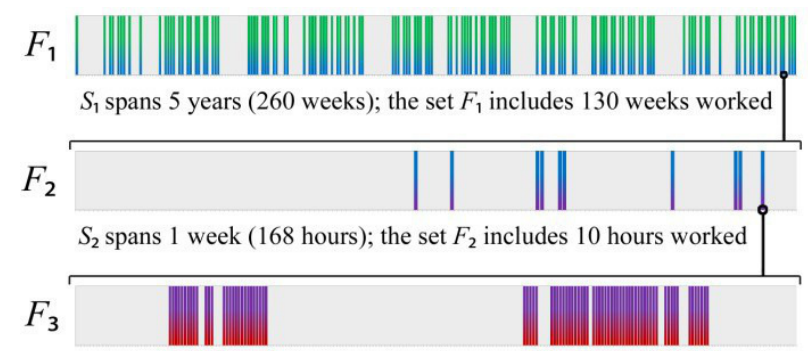

$S_{3}$ spans 60 minutes; the set $F_{3}$ includes 20 minutes worked

Fig. 2: Human Manager B's periods of work and non-work

\section{Temporal Dynamics of Artificial Agent Manager A}

Next consider a hypothetical Artificial Agent Manager A in the form of a software program running on a computer with a typical serial processor architecture. We suppose that during a given five-year operating period, there may be brief service outages for scheduled maintenance or updates but that there are no extended outages $\left(S_{1}=260\right.$ weeks, $N_{\delta}\left(F_{1}\right)$ $=260$ weeks). Each week, there is a scheduled maintenance window of one hour, when software updates are applied and the system is rebooted ( $S_{2}=168$ hours, $N_{\delta}\left(F_{2}\right)=167$ hours). The software program and hardware substrate for Artificial Agent Manager A have no non-work-related functions and

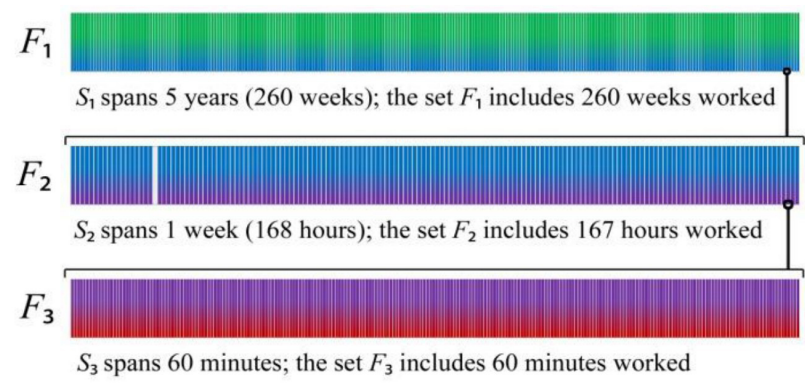

Fig. 3: Artificial Agent Manager A's periods of work and non-work

are not capable of being 'distracted' in the way that a human manager is, thus during a typical hour period of work, Artificial Agent Manager A does not dedicate any minutes to non-work-related functions $\left(S_{3}=60\right.$ minutes, $N_{\delta}\left(F_{3}\right)=60$ minutes). A graph of the temporal work dynamics of Artificial Agent Manager A is seen in Fig. 3 below. For an agent with these characteristics, we have calculated $D=$ $0.999, H=0.001$, and $A=99.4 \%$.

\section{Temporal Dynamics of Artificial Agent Manager B}

Finally, consider the hypothesized future scenario of Artificial Agent Manager B, an artificial general intelligence with a distributed neural network architecture that is modeled on the human brain and displays human-like motivations, emotions, and learning capacity [21]. While Artificial Agent Manager B enjoys its job, every two years it must spend a week away from work for a period of psychological assessment, maintenance, and relaxation, to reduce the likelihood of professional burnout $\left(S_{1}=260\right.$ weeks, $N_{\delta}\left(F_{1}\right)=258$ weeks). Moreover, during each week of work, its neural network architecture requires it to spend two hours daily in a 'sleep' mode in which any new external stimuli are shut out, in order to facilitate the assimilation of the day's experiences into long-term memory. In order to maintain its capacity for creativity, satisfy its intellectual curiosity, and avoid the development of cyberpsychoses, it must also spend two hours daily exploring spheres of experience unconnected to its work-related tasks $\left(S_{2}=168\right.$ hours, $N_{\delta}\left(F_{2}\right)=126$ hours). Because Artificial Agent Manager $B$ reflects the full constellation of human-like cognitive and social behaviors, it spends five minutes of each hour on functions other than work, such as

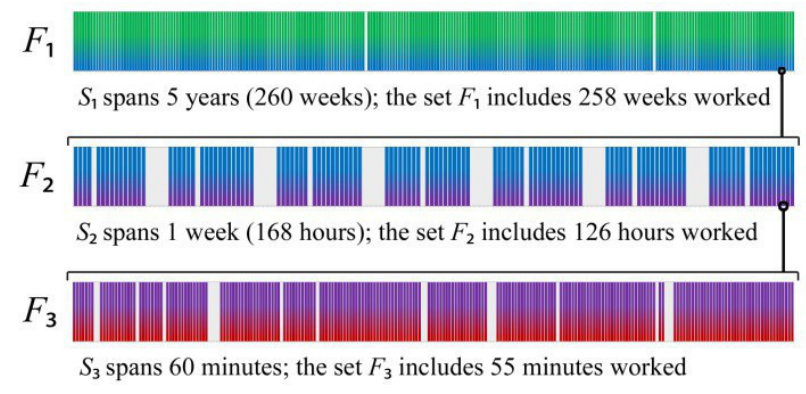

Fig. 4: Artificial Agent Manager B's periods of work and non-work 
TABLE II.

AGENTS' WORK EFFORT AS CHARACTERIZED BY FRACTAL DIMENSION, HuRST EXPONENT, AND AVAILABILITY

\begin{tabular}{|l|l|l|l|}
\hline \multicolumn{1}{|c|}{ Agent } & \multicolumn{1}{c|}{$\boldsymbol{D}$} & \multicolumn{1}{c|}{$\boldsymbol{H}$} & \multicolumn{1}{c|}{$\boldsymbol{A}$} \\
\hline Artificial Agent Manager A & 0.999 & 0.001 & $99.4 \%$ \\
\hline Human Manager A & 0.962 & 0.038 & $49.1 \%$ \\
\hline Artificial Agent Manager B & 0.945 & 0.055 & $68.2 \%$ \\
\hline Human Manager B & 0.532 & 0.468 & $1.0 \%$ \\
\hline
\end{tabular}

cyberloafing, following news stories, and communicating with friends ( $S_{3}=60$ minutes, $N_{\delta}\left(F_{3}\right)=55$ minutes).

A graph of the temporal work dynamics of Artificial Agent Manager B is seen in Fig. 4 below. For an agent with these characteristics, we have calculated $D=0.945, H=$ 0.055 , and $A=68.2 \%$.

\section{ANALYSIS AND Discussion}

\section{A. Comparison and Analysis}

Table II below gives the values of $D, H$, and $A$ for all four agents, ranked from the highest value of $D$ to the lowest.

We may note the following conclusions:

1) Artificial Agent Managers A and B and Human Manager $\mathrm{A}$ all display similar values of $H \approx 0$ (antipersistence), while Human Manager B displays a value of $H \approx 1 / 2$ (randomness). While more study is required to verify this supposition, it seems likely that managers with low persistence (as understood in the mathematical sense defined above) would be free from high switch costs, as their work intervals last longer, and they spend a smaller share of their work time transitioning into or out of periods of work.

2) The managers displaying high values for $D$ possess 'flexibility' in the sense that they are ready and available to work in almost every possible moment. However, they may simultaneously display 'inflexibility,' in the sense that they are used to working in every possible moment, thus unexpected interruptions may be more likely to derail the work of this sort of manager. Meanwhile, managers with a lower value for $D$ possess 'flexibility,' insofar as they are already used to working only sporadically and juggling intervals of work amidst many other activities, thus unexpected interruptions to their work may not greatly faze them. On the other hand, they might simultaneously display 'inflexibility,' insofar as the bulk of their time may already be filled with non-work-related activity, leaving only brief, sporadic slivers of time available for work. If an unexpected distraction prevents them from working during one of these windows, it may be quite some time before another window of availability for work appears.

3 ) The values of $A$ and $D$ are neither directly nor inversely proportional to one another. Artificial Agent Manager A possesses the highest values for both $A$ and $D$, while Human Manager B displays the lowest values for both. However, in the middle of the table, Human Manager A displays a higher value for $D$ than Artificial Agent Manager B but a lower value for $A$. This means that if one only utilizes a simple measure such as availability in assessing (and ranking) the temporal work dynamics of human and artificial agents, one will miss out on additional information that the fractal dimension and Hurst exponent can provide. While availability is a useful measure, it can potentially be misleading if not complemented by more sophisticated measures such as fractal dimension.

\section{B. Avenues for Future Research}

Further steps that we have identified to advance this research include:

1) Gathering empirical data about temporal work dynamics from a sample of real-world human managers and artificial agent systems to verify the appropriateness and value of this fractal-dimension-based model. Analysis of such data could aid in predicting the temporal dynamics of future artificial agent systems (for which empirical data is not yet available) and designing more advanced artificial intelligence systems that will be capable of carrying out a wider range of business management roles.

2) Adding data for a time-scale $S_{4}$ that captures the work activity of human and artificial agent managers as viewed in intervals as small as 10 milliseconds. The ability to capture such data for the neural activity of a human manager exceeds the temporal resolution available with current fMRI technology, but it may be possible using EEG or MEG techniques (perhaps in conjunction with fMRI).

3) Attempting to identify correlations between the values of $D$ and $H$ for a particular manager's temporal dynamics and traits identified in established models of managerial motivation and behavior.

In conclusion, we hope that if this paper's proposal for a single fractal temporal measure of work effort that is applicable to both human and artificial agent managers proves useful, it might in some way contribute to the development of a new perspective in which an organization's human resources management and its management of artificial agent systems are seen not as two disconnected spheres, but rather as two aspects of a new, integrated discipline of human and artificial agent resource management.

\section{REFERENCES}

[1] M. Williams, "Robot social intelligence," in Social Robotics, Lecture Notes in Computer Science 7621, 2012, pp. 45-55, http://dx.doi.org/10.1007/978-3-642-34103-8_5.

[2] M. Rehm, Y. Nakano, E. André, T. Nishida, N. Bee, B. Endrass, M. Wissner, A. A. Lipi, and H. Huang, "From observation to simulation: generating culture-specific behavior for interactive systems," in $A I$ \& Society, vol. 24, no. 3, pp. 267-80, October 1, 2009, http://dx.doi.org/ 10.1007/s00146-009-0216-3.

[3] M. Nunes and H. O'Neill, "Assessing the performance of virtual teams with intelligent agents," in Virtual and Networked Organizations, Emergent Technologies and Tools, Communications in Computer and Information Science 248, 2012, pp. 62-69,[3] http://dx.doi.org/10.1007/978-3-642-31800-9_7.

[4] R. L. Daft, Management, $10^{\text {th }}$ edition. Stamford, CT: Cengage Learning, 2011, pp. 7-8.

[5] Full-Time Equivalent (FTE). European Commission - Eurostat [Online]. Available [5] http://epp.eurostat.ec.europa.eu/ statistics_explained/index.php/Glossary:Full-time_equivalent.

[6] M. Grottke, H. Sun, R. M. Fricks, and K. S. Trivedi, "Ten fallacies of availability and reliability analysis," in Service Availability, Lecture Notes in Computer Science 5017, pp. 187-206, 2008, http://dx.doi.org/ 10.1007/978-3-540-68129-8_15. 
[7] N. J. Gunther, "Time - the zeroth performance metric," in Analyzing Computer System Performance with Perl::PDQ, pp. 3-46, Berlin: Springer, 2005, http://dx.doi.org/10.1007/978-3-540-26860-4_1.

[8] J. E. Y. Rossebeø, M. S. Lund, K. E. Husa, and A. Refsdal, "A conceptual model for service availability," in Quality of Protection, Advances in Information Security 23, pp. 107-18, 2006, http://dx.doi.org/10.1007/978-0-387-36584-8 9.

[9] Cool solutions: uptime workhorses: still crazy after all these years. (January 12, 2006). Novell Cool Solutions [Online]. Available http://www.novell.com/coolsolutions/trench/241.html.

[10] T. Tamai and Y. Torimitsu, "Software lifetime and its evolution process over generations," in Proceedings of 1992 Conference on Software Maintenance, pp. 63-69, 1992, [10] http://dx.doi.org/ 10.1109/ICSM.1992.242557.

[11] Employee tenure summary. (September 18, 2012). United States Department of Labor, Bureau of Labor Statistics [Online]. Available http://www.bls.gov/news.release/tenure.nr0.htm.

[12] Annual Hours Worked. Organisation for Economic Co-operation and Development [Online]. Available [12] http://www.oecd.org/els/emp/ ANNUAL-HOURS-WORKED.pdf.

[13] L. Golden, "A brief history of long work time and the contemporary sources of overwork," in Journal of Business Ethics, vol. 84, no. 2, pp. 217-27, Jan. 1, 2009, http://dx.doi.org/10.1007/s10551-008-9698-z.

[14] The Truth about the Billable Hour. Yale Law School [Online]. Available[14] http://www.law.yale.edu/studentlife/cdoadvice truthaboutthebillablehour.htm.

[15] J. Shih, "Project time in Silicon Valley," in Qualitative Sociology, vol. 27, no. 2, pp. 223-45, June 1, 2004, http://dx.doi.org/ 10.1023/B:QUAS.0000020694.53225.23.

[16] C. Sellberg and T. Susi, "Technostress in the office: a distributed cognition perspective on human-technology interaction," in Cognition, Technology \& Work, vol. 16, no. 2, pp. 187-201, May 1, 2014, http://dx.doi.org/10.1007/s10111-013-0256-9.

[17] A. Gupta, R. Sharda, and R. A. Greve, "You've got email! Does it really matter to process emails now or later?", in Information Systems Frontiers, vol. 13, no. 5, pp. 637-53, November 1, 2011, http://dx.doi.org/10.1007/s10796-010-9242-4.

[18] A. Hershatter and M. Epstein, "Millennials and the world of work: an organization and management perspective," in Journal of Business and Psychology, vol. 25, no. 2, pp. 211-23, June 1, 2010, http://dx.doi.org/10.1007/s10869-010-9160-y.

[19] F. Gobbo and M. Vaccari, "The Pomodoro Technique for sustainable pace in extreme programming teams," in Agile Processes in Software Engineering and Extreme Programming, Lecture Notes in Business Information Processing 9, pp. 180-84, 2008, [19] http://dx.doi.org/ 10.1007/978-3-540-68255-4_18

[20] R. Kurzweil, The Singularity Is Near: When Humans Transcend Biology. New York: Penguin Books, 2006. Kindle version, locations 2080-2120.

[21] J. Friedenberg, Artificial Psychology: The Quest for What It Means to Be Human. Psychology Press, 2011, pp. 24-26, 179-91, 199-200.

[22] A. Llarena, "Here comes the robotic brain!", in Trends in Intelligent Robotics, Communications in Computer and Information Science 103, pp. 114-21, 2010, http://dx.doi.org/10.1007/978-3-642-15810-0_15.

[23] J. L. McClelland, "Is a machine realization of truly human-like intelligence achievable?", in Cognitive Computation, vol. 1, no. 1, pp. 17-21, March 1, 2009, http://dx.doi.org/ 10.1007/s12559-009-9015-x.

[24] R. Kurzweil, The Age of Spiritual Machines: When Computers Exceed Human Intelligence. New York: Penguin Books, 2000. Kindle version, location 1754.

[25] L. F. Abbott and P. Dayan, Theoretical Neuroscience: Computational and Mathematical Modeling of Neural Systems. Cambridge: MIT Press, 2001. As cited in A. Llarena, "Here comes the robotic brain!", in Trends in Intelligent Robotics, Communications in Computer and Information Science 103, pp. 114-21, 2010, [25] http://dx.doi.org/ 10.1007/978-3-642-15810-0_15
[26] S. W. Brown and S. M. Merchant, "Processing resources in timing and sequencing tasks," in Perception \& Psychophysics, vol. 69, no. 3, pp. 439-49, April 1, 2007, http://dx.doi.org/10.3758/BF03193764.

[27] T. D. Wager, J. Jonides, and E. E. Smith, "Individual differences in multiple types of shifting attention," in Memory \& Cognition, vol. 34, no. 8, pp. 1730-43, December 1, 2006, [27] http://dx.doi.org/ 10.3758/BF03195934

[28] M. C. Schippers and R. Hogenes, "Energy management of people in organizations: a review and research agenda," in Journal of Business and Psychology, vol. 26, no. 2, pp. 193-203, [28] http://dx.doi.org/ 10.1007/s10869-011-9217-6.

[29] P. U. Tse, J. Intriligator, J. Rivest, and P. Cavanagh, "Attention and the subjective expansion of time," in Perception \& Psychophysics, vol. 66, no. 7, pp. 1171-89, October 1, 2004, [29] http://dx.doi.org/ $10.3758 / \mathrm{BF} 03196844$

[30] G. Marchetti, "Observation levels and units of time: a critical analysis of the main assumption of the theory of the artificial," in AI \& Society, vol. 14, no. 3-4, pp. 331-47, September 1, 2000, [30] http://dx.doi.org/ 10.1007/BF01205515.

[31] W. Levelt, "Models of word production," in Trends in Cognitive Sciences, vol. 3, no. 6, pp. 223-32, 1999, [31] http://dx.doi.org/10.1016/ S1364-6613(99)01319-4.

[32] C. Hamilton, Essentials of Public Speaking. Stamford, CT: Cengage Learning, 2014, p. 60

[33] M. J. Traxler, Introduction to Psycholinguistics: Understanding Language Science. Hoboken, NJ: John Wiley \& Sons, 2011, Ch. 10.

[34] World's fastest typer. Chicago Tribune [Online]. Available http://www.chicagotribune.com/sns-viral-fastest-records-pictures018,0,193476.photo.

[35] "New World's Record for Shorthand Speed: Nathan Behrin Transcribed 350 Words in a Minute With Only Two Errors," New York Times, December 30, 1922.

[36] Fastest talker. Guinness World Records [Online]. Available http://gwrstaging.untitledtest.com/world-records/1/fastest-talker.

37] G. Longo and M. Montévil, "Scaling and scale symmetries in biological systems," in Perspectives on Organisms, Lecture Notes in Morphogenesis, Berlin: Springer, 2014, pp. 23-73, http://dx.doi.org/10.1007/978-3-642-35938-5 2.

[38] D. Lloyd, "Biological time is fractal: early events reverberate over a life time," in Journal of Biosciences, vol. 33, no. 1 pp. 9-19, March 1, 2008, http://dx.doi.org/10.1007/s12038-008-0017-8.

[39] O. Sourina, Q. Wang, Y. Liu, and M. K. Nguyen, "Fractal-based brain state recognition from EEG in human computer interaction," in Biomedical Engineering Systems and Technologies, Communications in Computer and Information Science 273, pp. 258-72, 2013 , http://dx.doi.org/10.1007/978-3-642-29752-6_19.

[40] B. B. Mandelbrot, The Fractal Geometry of Nature. London: Macmillan, 1983, pp. 353-54

[41] J. M. Valverde, A. Castellanos, and M.A.S. Quintanilla, "Looking for memory in the behavior of granular materials by means of the Hurst analysis," in Of Stones and Man: From the Pharaohs to the Present Day, J. Kerisel, Ed. London: Taylor \& Francis Group, 2005, pp. 817.

[42] L. Telesca, V. Cuomo, V. Lapenna, and M. Macchiato, "On the methods to identify clustering properties in sequences of seismic timeoccurrences," in Journal of Seismology, vol. 6, no. 1, pp. 125-34, January 1, 2002, http://dx.doi.org/ 10.1023/A:1014275509447.

[43] Y. Zhang, Q. Li, W. Chu, C. Wang, and C. Bai, "Fractal structure and fractal dimension determination at nanometer scale," in Science in China Series A: Mathematics, vol. 42, no. 9, pp. 965-72, September 1, 1999, http://dx.doi.org/10.1007/BF02880388.

[44] K. Falconer, Fractal Geometry: Mathematical Foundations and Applications. Hoboken, NJ: John Wiley \& Sons, 2004, pp. 41-44

[45] B. Wahl, P. Van Roy, M. Larson, and E. Kampman, Exploring Fractals on the Macintosh. Reading, MA: Addison-Wesley Professional, 1994, pp. 75-108. 